\title{
Miranda
}

Revue pluridisciplinaire du monde anglophone /

Multidisciplinary peer-reviewed journal on the English-

speaking world

$11 \mid 2015$

Expressions of Environment in Euroamerican Culture /

Antique Bodies in Nineteenth Century British

Literature and Culture

\section{Notre Top 8 des films anglophones de 2014}

David Roche et Vincent Souladié

\section{OpenEdition}

1 Journals

Édition électronique

URL : https://journals.openedition.org/miranda/7441

DOI : 10.4000/miranda.7441

ISSN : 2108-6559

Éditeur

Université Toulouse - Jean Jaurès

Référence électronique

David Roche et Vincent Souladié, « Notre Top 8 des films anglophones de 2014 », Miranda [En ligne],

11 | 2015, mis en ligne le 21 juillet 2015, consulté le 08 septembre 2022. URL : http://

journals.openedition.org/miranda/7441; DOI : https://doi.org/10.4000/miranda.7441

Ce document a été généré automatiquement le 8 septembre 2022

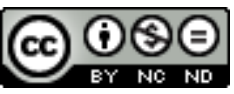

Creative Commons - Attribution - Pas d'Utilisation Commerciale - Pas de Modification 4.0 International - CC BY-NC-ND 4.0

https://creativecommons.org/licenses/by-nc-nd/4.0/ 


\title{
Notre Top 8 des films anglophones de 2014
}

\author{
David Roche et Vincent Souladié
}

1 N'en déplaise à ceux qui ne cessent de proclamer la mort du cinéma, 2014 aura été une grande année pour le 7e Art - Ida (Pologne/Danemark/France/UK, Pawel Pawlikowski), Mommy (Canada, Xavier Dolan), Winter Sleep (Turquie/France/Allemagne, Nuri Bilge Ceylan) - et notamment anglophone. La liste s'étend si on inclut des films de 2013 sortis sur les écrans français en début d'année: Her (USA, Spike Jonze), Nymphomaniac (Danemark/ Allemagne/Belgique/UK/France, Lars Von Trier), Only Lovers Left Alive (UK/Allemagne/France/Grèce/Chypre, Jim Jarmusch) ou encore Mister Babadook (Australie/Canada, Jennifer Kent), récompensé au Festival du film fantastique de Gérardmer 2014. Face à une concurrence si rude, certains films très réussis ont été écartés : Guardians of the Galaxy (USA/UK, James Gunn), Foxcatcher (USA, Bennett Miller), mélange de deux genres hollywoodiens classiques (le biopic et le film de catch) dans lequel l'humoriste Steve Carell livre une prestation à la fois abjecte et pathétique, et Gone Girl (USA, David Fincher), thriller doté d'un récit solide et servi par un casting convaincant (Rosamund Pike en particulier a fait l'unanimité). Hormis quelques coups de cœurs, notre liste n'est finalement pas très originale et comprend des films primés à Cannes, aux BAFTAs et aux Oscars. Certains films - Birdman, Interstellar - ont néanmoins eu une réception critique mitigée, notamment en France.

\section{Birdman: Or, (The Unexpected Virtue of Ignorance) (USA, Alejandro González Iñárritu)}

2 Cinquième film du cinéaste mexicain, Oscar 2015 du meilleur film et de la mise en scène, Birdman s'inscrit dans la grande tradition du métafilm hollywoodien, de A Star is Born (William Wellman et Jack Conway, 1937), All About Eve (Joseph L. Mankiewicz, 1950) et Sunset Blvd. (Billy Wilder, 1950), à Mulholland Drive (David Lynch, 2001). Ancré dans une opposition cinéma/théâtre, Hollywood/Broadway, Birdman est un peu la synthèse des deux grands films de Mankiewicz et Wilder : un acteur connu pour une série de 
films de super héros veut faire son come-back en s'affirmant comme acteur et metteur en scène, et donc comme un artiste à part entière. Le casting de Michael Keaton, le Batman de Tim Burton $(1989,1992)$, est, à ce titre, central. Le choix d'adapter la nouvelle «Parlez-moi d'amour » ("What We Talk About When We Talk About Love », 1978) de Raymond Carver est également pertinent. En effet, il en dit long sur le personnage de Riggan : si celui-ci s'identifie à la nouvelle du fait que son père était alcoolique, son ambition transparaît dans le choix d'adapter une œuvre réputée inadaptable, tandis que le titre de la nouvelle renvoie au narcissisme des acteurs et creuse l'écart entre l'Amérique ordinaire dépeinte par Carver et la scène de Broadway. On peut reprocher à Birdman le traitement quelque peu convenu de la confrontation entre Riggan et la critique new-yorkaise, mais la performance des acteurs reste tout simplement impressionnante, notamment quand ils ne cessent d'entrer et de sortir de leurs rôles fictifs (mention spéciale pour la confrontation Michael Keaton / Edward Norton). Le film contient par ailleurs des fulgurances, telle que l'envol final du Birdman comme réécriture sublime du moment de streaking de Riggan.

\section{Boyhood (USA, Richard Linklater)}

3 En contraste avec l'ambition inédite et expérimentale de son dispositif narratif, fictionnaliser douze ans d'une vie humaine filmée en temps réel à raison d'une semaine de tournage par an, Boyhood peut surprendre par la modestie et la douceur de son regard. Linklater refuse de brasser les années de vie de son jeune héros avec l'autorité d'une fresque monumentale. Son cinéma fait au contraire le pari de l'accident et du superflu ; il s'accorde le temps de laisser sourdre de chaque instant volatile des amorces de gravité toujours réversibles dans la candeur. Linklater trouve en fait matière à croiser et à étoffer les deux principales lignes de force de son cinéma : l'errance douceamère de l'adolescence périurbaine américaine résignée à la mélancolie de ses rituels (Slacker, 1991; Dazed and Confused, 1993), et le long étagement temporel des relations humaines (le triptyque Before Sunrise, 1995, Before Sunset, 2004, Before Midnight, 2013). Devant la suite de micro-récits plus ou moins déliés de Boyhood, emportés par un tempo indolent, chacun sauvegardera pour lui du flux d'images ses propres pincements d'émotions à la reconnaissance soudaine d'un vécu partagé, que se soit des fétiches matérialistes de l'histoire technologique d'une génération ou des fragments d'expériences existentielles. Nous retiendrons particulièrement le travail de l'abandon vécu par des acteurs qui ont choisi de s'en remettre à la confiance de la caméra pour lui livrer, mise à nue par le resserrement temporel du montage, l'évolution physique imprévisible de leur corps et de leurs traits. Le projet esthétique de Linklater tient sur ce principe : la fiction s'acclimate d'une réalité sans programme et se laisse suivre comme un documentaire en creux.

\section{Calvary (Ireland/UK, John Michael McDonagh)}

4 Le réalisateur anglo-irlandais John Michael McDonagh construit le récit fataliste d'un prêtre solitaire gratuitement condamné à mort, dès la première séquence du film, par une de ses ouailles assoiffée de vengeance pour avoir subi enfant les abus d'un homme d'église. Le prêtre bénéficie d'une dernière semaine de liberté avant son exécution programmée, un délai tragique qui échelonne le récit, lors duquel il essaie de mobiliser 
les habitants de sa paroisse mais voit tous ses adjuvants potentiels lui tourner le dos. Avec une noirceur tragique nuancée d'un humour grinçant, McDonagh dépeint la solitude d'une vie pieuse et sacrificielle tourmentée par le déclin collectif de la foi. Après avoir passé la formule de l'actionner hollywoodien au filtre de la comédie sociale anglaise dans L'Irlandais (2011), c'est avec un pseudo remake du Train Sifflera Trois Fois (Fred Zinnemann, 1952) que le cinéaste investit les paysages côtiers irlandais et se plait à filmer le gigantesque décor rocheux du comté de sligo comme un Monument Valley local, invoquant les cathédrales naturelles de l'imaginaire fordien (célèbre irlandoaméricain) comme cadre à un récit sur la foi. McDonagh conserve du western des axes dramatiques, la morale, la filiation et l'attachement au territoire, ainsi que ce sens lyrique du milieu englobant, prouvant combien ce genre est toujours apte à se réinventer avec originalité hors des balises spatiales et temporelles officiellement assignées.

\section{The Grand Budapest Hotel (USA/Germany/UK, Wes Anderson)}

5 On a pu reprocher à Anderson le côté anhistorique ou décontextualisé de ses films même si cela semble quelque peu infondé concernant $L a$ famille Tennenbaum (2001). Se déroulant dans un pays imaginaire, la république de Zubrowka, son huitième film maintient l'atmosphère féérique de ses œuvres précédentes mais propose, sur fond de montée du nazisme, une leçon de tolérance à travers un homme médiocre (M. Gustave) qui s'opposera avec dignité aux valeurs du Reich. On retrouve les thèmes de prédilection d'Anderson (la famille, la filiation), ainsi que ses fameux plans frontaux, seulement la lenteur de ses films précédents fait place à un rythme frénétique emprunté au film d'espionnage. L'éblouissement est total: costumes et décors somptueux et partition originale magnifique, justement récompensés aux Oscars; interprétation parfaite du tandem Ralph Fiennes / Tony Revolori, mais aussi des rôles secondaires, à commencer par un Willem Dafoe dont le look évoque un curieux mélange entre les scélérats de l'expressionisme allemand et le Requin de L'Espion qui m'aimait (Lewis Gilbert, 1977). On se demandait déjà après Moonrise Kingdom (2012) si Anderson réussirait à dépasser ce sommet. The Grand Budapest Hotel est la preuve qu'il possède des ressources insoupçonnées, faisant de lui le cinéaste américain de sa génération dont l'œuvre est la plus impressionnante.

\section{Interstellar (USA/UK/Canada, Christopher Nolan)}

6 Le huitième long-métrage du cinéaste britannique n'est peut-être pas un film parfait, mais il s'avère un film ambitieux qui attire immédiatement l'attention sur un fait : le cinéma se risque rarement à faire de la science-fiction dure. Or, si certains ont pu contester l'exactitude du socle théorique du film, Christopher Nolan et son frère (qui cosigne le scénario) ont le mérite d'ancrer le récit tout entier dans la science: le personnage principal (Cooper) est un scientifique qui porte un regard informé sur son environnement ; les scènes d'action (dont la grande vague) sont également justifiées au niveau conceptuel; même le dilemme intérieur du personnage - la science ou la famille - est justifiée scientifiquement, puisque la force qui anime le personnage pour retrouver sa fille ne relève ni de son héroïsme, ni de normes sociales, mais de l'instinct 
paternel. En cela, le film se positionne clairement dans le sillage de 2001 (Stanley Kubrick, 1968) et de Solaris (Andrei Tarkovsky, 1972) et prend le contre-pied des blockbusters hollywoodiens; le simple fait que Murph se rende compte qu'il a fait le mauvais choix est, au niveau métafictionnel, une critique des films à la Armageddon (Michael Bay, 1998). Rien qu'à ce titre, ainsi que pour la beauté de ses images, Interstellar, comme le récent Gravity (Alfonso Cuarón, 2013), fait beaucoup de bien au genre.

\section{Nightcrawler (USA, Dan Gilroy)}

7 Ce premier film du scénariste américain est plus que prometteur : il est un véritable tour de force. Les premiers plans sur les abords nocturnes de Los Angeles annoncent le mariage formel de Michael Mann et de Martin Scorsese, de la ville sidérale à la ville décrépite où rôde un paria solitaire. Mais Gilroy n'insiste pas trop sur ces références esthétiques et s'en tient rapidement à dérouler un récit animé d'un souffle morbide. S'il situe son film dans l'univers des médias racoleurs, le cinéaste se montre moins intéressé par la critique somme toute évidente de la société du spectacle que par la figure-titre du sociopathe hérité de l'imaginaire cynique et dépressif de Bret Easton Ellis. Ce modèle lui permet de réinventer le personnage bien connu du chasseur de scoops sans scrupules, brillamment incarné ici par un Jack Gyllenhaal terrifiant aux antipodes de son rôle phare dans Brokeback Mountain (Ang Lee, 2005). Ce dernier confirme l'adoption radicale du method acting qui définit la nouvelle orientation de sa carrière depuis quelques films. Il prête à Louis Bloom des traits poisseux et un regard creusé, animés par la candeur glaciale de son éternel rictus, fondu dans les lumières de l'entité urbaine dont il incarne une nouvelle et inoubliable figure de cauchemar moderne.

\section{Under the Skin (UK/USA/Switzerland, Jonathan Glazer)}

Projeté lors de festivals en 2013, le troisième long-métrage du cinéaste britannique, connu pour ses clips de Radiohead, a été vendu et reçu comme un film de sciencefiction, avec Scarlett Johansson dans le rôle d'une extra-terrestre qui semble ingérer ses victimes humaines, un peu à la manière des vampires de Lifeforce (Tobe Hooper, 1985). L'esthétique du film joue sur un contraste saisissant entre les sublimes scènes de consommation hyper-stylisées, et les scènes de traque à l'approche quasi-documentaire (plusieurs des personnages masculins que rencontre le personnage féminin sont joués par des amateurs qui ignoraient qu'une caméra tournait). Or, cette esthétique dichotomique attire l'attention sur le fait qu'Under the Skin est un film-puzzle à la manière de Mulholland Dr. (David Lynch, 2001), qui, comme Videodrome (David Cronenberg, 1983), peut également être interprété comme le récit du délire du personnage principal, dont l'inhumanité serait finalement une couverture pour nier une pathologie sexuelle humaine; le titre du film invite également à cette double lecture. La force de ce long métrage réside donc dans le talent du cinéaste, du photographe et de l'actrice (à la fois inquiétante et vulnérable, et tout simplement impressionnante) à conjuguer les deux en un (la SF et le naturalisme, l'extra-terrestre ou la schizophrène), ce que Glazer avait déjà tenté dans son film précédent, Birth (2004). 
La scène de la plage est l'une des scènes les plus dures que nous avons pu voir ces dernières années.

\section{Whiplash (USA, Damien Chazelle)}

9 Très remarqué par la critique et le public, Whiplash n'est que le deuxième long métrage de Damien Chazelle et le premier distribué en France. La mise en scène y fait montre d'une rigueur classique appropriée au sujet : l'apprentissage méthodique de la métrique musicale par un aspirant batteur. Le film musical n'est pourtant qu'un prétexte propre à entraîner le récit d'initiation sur une voie des plus ambiguës. En effet, il explore les relations toxiques entre un professeur psychotique (J.K. Simmons, meilleur second rôle aux BAFTA et aux Oscars) et son élève le plus acharné, la pédagogie et l'abnégation prenant le pli d'une aliénation mentale dont le montage restitue la raideur. Peu inspiré par les vibrations du cinéma jazz hollywoodien, Young Man with a Horn (Michael Curtiz, 1950) ou The Man With the Golden Arm (Otto Preminger, 1955), Chazelle s'applique plutôt à démarquer l'ordre et la maitrise de David Fincher. S'il est évident que les effets tapeà-l'œil de la longue séquence finale cherchent à tenir l'émotion chevillée à la forme, c'est que l'enchaînement des plans s'articule à un rythme qui se veut l'expression autoritaire de la perfection. La prise de risque de cette ambiguïté morale entre la liberté de la composition et la rectitude de l'effort traduit chez Chazelle une belle confiance en ses propres outils expressifs.

\section{INDEX}

Keywords : American cinema, British cinema, Irish cinema, 2014, Birdman, Boyhood, Calvary, The Grant Budapest Hotel, Interstellar, Nightcrawler, Under the Skin, Whiplash

Mots-clés : cinéma américain, cinéma britannique, cinéma irlandais, 2014, Birdman, Boyhood, Calvary, The Grant Budapest Hotel, Interstellar, Nightcrawler, Under the Skin, Whiplash

Thèmes : Film

\section{AUTEURS}

\section{DAVID ROCHE}

Professor of Film Studies / professeur d'études filmiques

Université Toulouse Jean Jaurès

mudrock@neuf.fr

\section{VINCENT SOULADIÉ}

Docteur en études cinématographiques

Université Toulouse Jean Jaurès

vsouladi@univ-tlse2.fr 